\title{
EFFECT OF MIXER TYPE ON FRESH AND HARDENED PROPERTIES OF ULTRA-HIGH-PERFORMANCE CONCRETE (UHPC)
}

\author{
Ernests Ozolins $^{1}$, Juris Zavickis ${ }^{2}$, Arturs Lukasenoks ${ }^{1}$, Arturs Macanovskis ${ }^{3}$
}

${ }^{1}$ Betona pētijumu centrs, Latvia; ${ }^{2}$ Dzelzsbetons MB, Latvia; ${ }^{3}$ Riga Technical University, Latvia ernests.ozolins@bpcentrs.lv, juris.zavickis@mbbetons.lv, arturs.lukasenoks@ @rtu.lv, arturs.macanovskis@rtu.lv

\begin{abstract}
Ultra-high-performance concrete (UHPC) appears to be one of the key enabling construction materials of the 21 st century. The term UHPC concrete refers to a new class of advanced cementitious composite materials with superior mechanical and durability properties when compared to conventional concrete. The production method of UHPC requires effective mixing of highly structured active components (reactive cement, micro silica, fly-ash and others). The development of the complex mixture of UHPC concrete occurs in lab conditions with small batches and lab-sized mixers. However, once a mix has been developed the large-scale production has to be transferred into a factory-based production. This means that the batch size is significantly increased, as well as the mixer is not the same as the one used in laboratory-based trials. This research paper focuses on the observable differences of the fresh and hardened UHPC properties that occur when different mixers and batch sizes are used. Tests, such as slump flow test, time for slump flow to reach a certain diameter and compressive strength tests were used to evaluate the differences between different mixers and batch sizes. The breaking time of the mix - the time it takes for the mix to become visually fluid and wet, was also noted. It was found that not only is the chosen mixer an important factor when scaling up the production from laboratory-based to larger-scale production, but also the chosen superplasticizer can greatly affect the fresh, as well as the hardened properties of a UHPC mix, when different mixers and batch sizes are used. It was also found that increased mixing speed reduced the breaking time of the mix for recipes with a high slump flow value. This behaviour, however, was not noticed for mixes with lower slump flow value.
\end{abstract}

Keywords: UHPC, concrete mixers, rheology.

\section{Introduction}

Ultra-high-performance concrete (UHPC) is a relatively new development in the concrete industry, with the first designs developed in 1990 in France [1]. UHPC concrete refers to a new class of advanced cementitious composite materials characterized by its self-compacting capabilities, as well as a very high compressive strength and durability [2]. The high compressive strength of UHPC is obtained by the use of a low water-to-cement ratio, the addition of ultra-fine material such as micro silica and fly ash, and a high dosage of a superplasticizer [1;3-5]. Improper mixing can lead to variations of these important components throughout the matrix $[1 ; 5 ; 6]$. Because of the very high compressive strength and fine microstructure of the concrete, it is much more sensitive to defects in the matrix, which can result in reduced performance of concrete. Inhomogeneity of the mix can lead to reduced workability, segregation, and reduction in the compressive strength of concrete [4; 7].

The development of new concrete mixes, such as UHPC concrete, starts with small-scale laboratory trials for a quicker research process and more efficient use of resources. Once optimal properties have been obtained in the lab-based trials it is then necessary to up-scale the manufacturing process. Because of the differences in the batch size, mixing speed and mixer design, differences in the way the mix behaves in a laboratory and during larger-scale tests may occur. It is thus necessary to understand how changes in these aspects will affect the scaling up of UHPC manufacturing from laboratory sized mixers to larger-scale mixers.

The homogeneity of a concrete mix can be measured directly by determining the concrete constituent material distribution within the mix in various samples taken during the discharge process. It can also be measured indirectly, by measuring parameters related to the workability of the mix, such as the slump flow and the relative viscosity measured by the T20 time, and by comparing the sample compressive strength distribution between the samples taken [4; 7].

Two types of concrete mixers may be used for UHPC mixing - pan mixers or drum mixers. It has been shown that insufficient homogeneity is achieved when mixing with a drum mixer [8]. That is why pan mixers are chosen as the mixers of interest for this study. A pan mixer consists of a bowl-shaped container - pan, which holds the material together, then a single or series of blades, paddles spin to mix 
the concrete. Usually, a scraper can be found at the edge of the pan to scrape off any material that might have been stuck at the edge of the pan [6;7].

There have been four stages of UHPC mixing characterized [8].

1. Formation of granules right after the addition of water and superplasticizer to the mix. The granules are created by the fine material adhering to the liquid that has been introduced to the mix. Over time the granules keep growing bigger as additional fine particles are collected on the surface of the granules.

2. The second stage is reached when all of the fine powder-like material has been collected on the surface of the granules and the surface of the granules starts to appear wet.

3. The third stage can be called the breakdown stage. At this stage, the granules start to break down creating a paste-like substance. At this point, the mixture is not still homogeneous as some parts of the mixture may appear to be wetter than others.

4. At this stage all of the granules have been broken and a homogenous mixture has been achieved [8].

The mixing speed on its own can influence the rheological properties of the mix [1;9]. At the mixing speeds generally used in manufacturing, once the mixing speed is increased the yield stress and the plastic viscosity of the mix can be significantly reduced [5;10], increased mixing energy also increases the early hydration rate of concrete [6]. Changes in the batch size, as well as the structure of the pan mixer used, also affect the homogeneity of the final UHPC mix $[6 ; 8 ; 10]$. Changing the cement and cement replacement material amounts will also lead to different mixing requirements [6].

\section{Materials and methods}

Materials used in this study.

- Aalborg white cement as the primary binder (specific gravity of 3.15, mean particle diameter of $12.7 \mu \mathrm{m}$ and specific surface area of $\left.1676 \mathrm{~m}^{2} \cdot \mathrm{kg}^{-1}\right)$.

- Elkem micro silica 940U (Specific gravity - 2.2, mean particle diameter - $0.316 \mu \mathrm{m}$, a specific surface area greater than $\left.15 \mathrm{~m}^{2} \cdot \mathrm{g}^{-1}\right)$.

- Punkte test optimized mix of 4 quartz fractions from our previous study with a specific gravity of 2.612 and maximum grain size of $1.25 \mathrm{~mm}$ [11].

- Acrylic polymer-based superplasticizer (SP) with $30 \%$ solid content by Stachema (SP2).

- Acrylic polymer-based superplasticizer (SP) with 30\% solid content - Mapei Dynamon SX-130 (SP1).

- Tap water.

Three different dosages of micro silica were trialled for both of the SPs in this study. As it is shown in Table 1, the dosage of the SP of $1.5 \%$ (wtob) and water to binder ratio of 0.25 were kept constant throughout the study. The dosage of the optimized quartz aggregate was altered with increasing the MS dosage as shown in the table below.

Table 1

Mix constituents of the samples tested

\begin{tabular}{|c|c|c|c|c|}
\hline Sample ID & Cement, $\mathrm{kg} \cdot \mathrm{m}^{-3}$ & Micro silica, $\mathrm{kg} \cdot \mathrm{m}^{-3}$ & $\begin{array}{l}\text { Optimized quartz } \\
\text { fraction, } \mathrm{kg} \cdot \mathrm{m}^{-3}\end{array}$ & SP used \\
\hline SP1-00 & 800 & 0 & 1380.2 & SP1 \\
\hline SP1-40 & 760 & 40 & 1365.9 & SP1 \\
\hline SP1-80 & 720 & 80 & 1351.6 & SP1 \\
\hline SP2-00 & 800 & 0 & 1380.2 & SP2 \\
\hline SP2-40 & 760 & 40 & 1365.9 & SP2 \\
\hline SP2-80 & 720 & 80 & 1351.6 & SP2 \\
\hline
\end{tabular}

An optimized version of the mix SP2-40 was trialled on all of the mixers tested in this study. The optimized version SP2-40P had the superplasticizer dosage reduced to $1.03 \%$ (wtob) in order to increase the viscosity of the mix. 
For precise measurements, scales (LW Measurements HRB series scales) with the accuracy of up to $0.1 \mathrm{~g}$ were used to weigh quartz, cement and MS. Scales with a precision of up to $0.01 \mathrm{~g}$ (SF-400C) were used to weigh water and SP for tests.

Three different mixers were used in this trial as shown in Table 2 . The only mix that had been tested with the mixer M3 for its fresh properties was the mix SP2-40P. Mixers M2 and M3 are classified as counter-current motion pan mixers [7]. For the mixer M1 a certain classification has not been given.

Table 2

Mixers used in the study

\begin{tabular}{|c|c|c|c|c|}
\hline Mixer ID & Active element & Revolutions per minute & $\begin{array}{c}\text { UHPC mixing } \\
\text { capacity, L }\end{array}$ & Samples made \\
\hline M1 & Mixing head & 135.5 & 1 & All \\
\hline M2 & Mixing container & 41 & 30 & All \\
\hline M3 & Mixing paddles & 35 & 150 & SP2-40P \\
\hline
\end{tabular}

Mixer M1 is a laboratory-grade mixer with a capacity to mix approximately 1 litre of UHPC and 5 litres of conventional concrete. The active element of the mixer is the mixing head, which is moving at 135.5 revolutions per minute. For this mixer, the mixing container is stationary during the mixing procedure.

a)

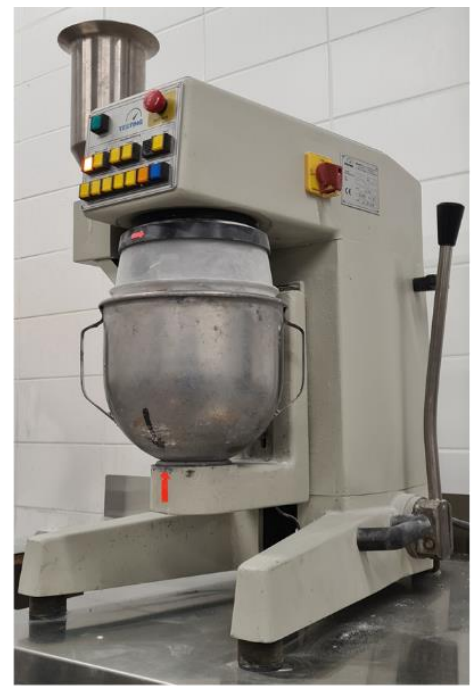

b)

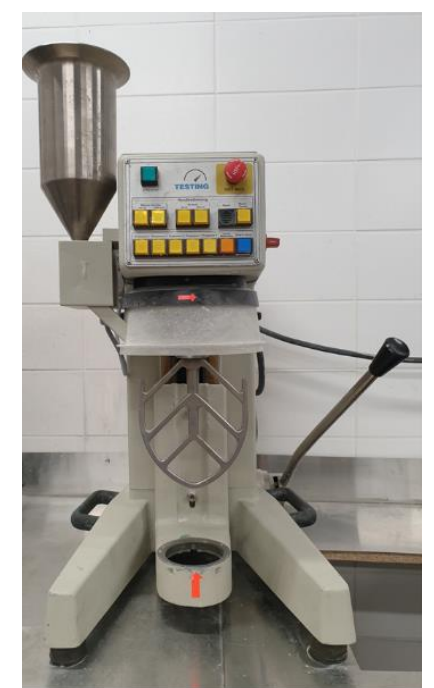

Fig. 1. Mixer M1 with the mixing bowl (a) showing the mixer head with bowl removed (b)

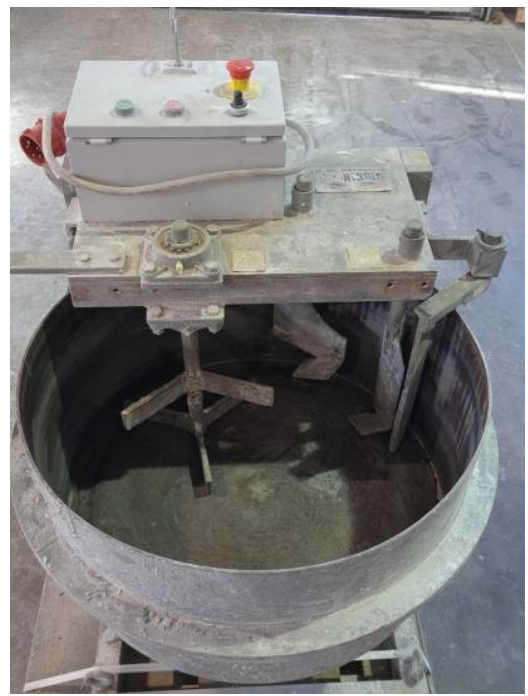

Fig. 2. Mixer M2 
Mixer M2 has a capacity to mix $60 \mathrm{~L}$ of conventional concrete, however, the maximum capacity is halved for UHPC $-30 \mathrm{~L}$ with the minimum capacity being $10 \mathrm{~L}$. The element that is moving for this mixer is the mixing container, which is rotating around at a speed of 41 revolutions per minute and the mass within the container is then moving the mixing pedals.
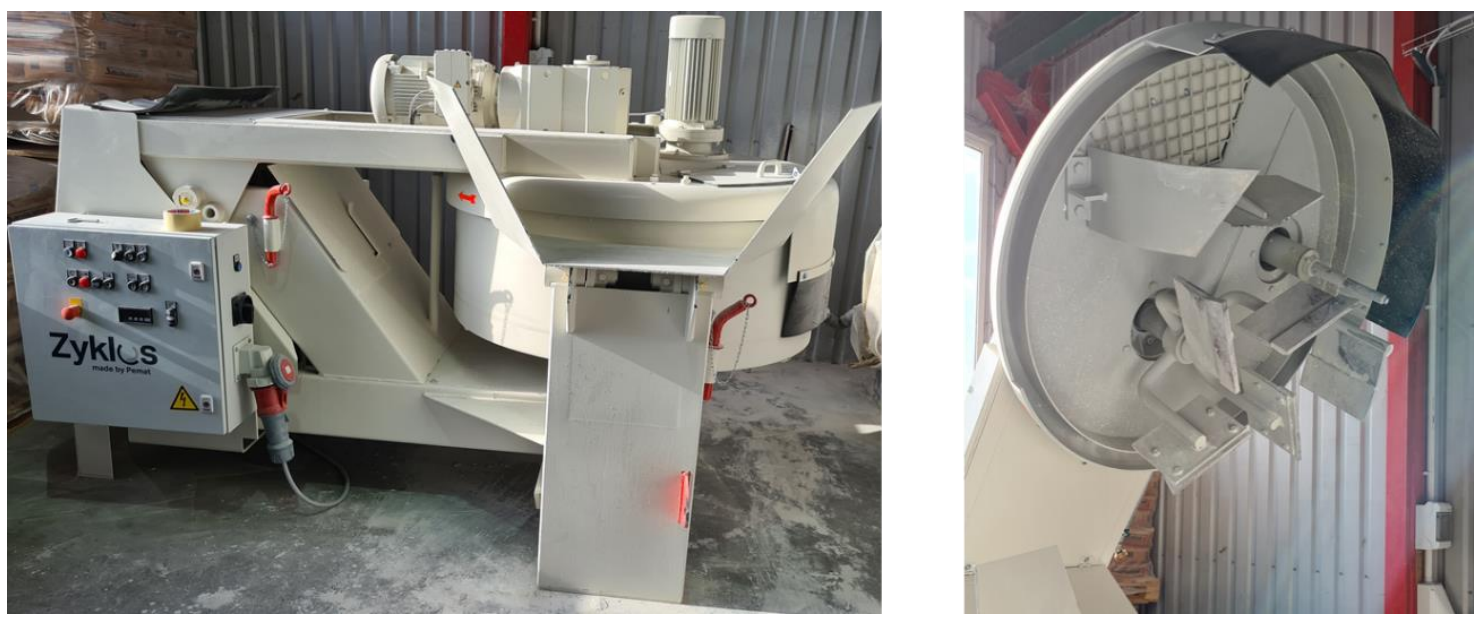

Fig. 3. Mixer M3

Mixer M3 is a mixer with a maximum capacity to mix $150 \mathrm{~L}$ and a minimum capacity of $70 \mathrm{~L}$ of UHPC. In this mixer, the mixing pedal head is the element that is moving. The movement speed of the mixing pedal head is adjustable for this mixer. The mixing container is free moving and during the mixing process moves together with the mixing pedals due to the friction of the mass. During this study, a mixing speed of 35 revolutions per minute was used throughout the mixing process.

In the mixer M1 0.6 litres of UHPC were made for each batch of tests. The produced amount of UHPC was sufficient for a slump flow test and enough to create two prism samples for compressive strength testing. For UHPC made with the M2 mixer the batch size was 21 litres. For the mix made with the M3 mixer the batch size was 70 litres. For all of the mixers, the mixing procedure was the same - all four sand fractions are put in the mixer first and mixed until a homogeneous mix is obtained. Then cement and MS are added to the mixer and it is further mixed for another 2 minutes. After that, a mixture of water and the required admixture dosage is poured into the mixer. Concrete is then mixed further for 10 more minutes.

The slump flow test was done in accordance with the ASTM C230 standard. The time it takes for concrete to reach a slump of $20 \mathrm{~cm}$ in diameter, T20 time, as well as the maximum slump flow diameter, Dmax, were measured.

Samples for compressive strength testing made with the M1 mixer were made using prisms with dimensions 40x40x160 mm, concrete was poured in one go and no additional compaction was done. From each testing batch, 2 prisms were made. Before compressive strength tests, the prism samples were cut into 3 pieces each with dimensions of $40 \times 40 \times 40 \mathrm{~mm}$. For the samples made with the M2 mixer, the samples for testing were made using metallic cube moulds with dimensions $100 \times 100 \times 100 \mathrm{~mm}$. A total of 6 cube samples were made. The curing and compressive strength, and density measurements of the samples were done in accordance with the EN 12390 standard. Half of the samples were tested at 7 days of age, and the remaining samples were tested at the age of 28 days. In order to compare results between $40 \mathrm{~mm}$ and $100 \mathrm{~mm}$ cubes, a scaling factor of 1.15 was used to level the strength gain from the smaller size of the $40 \mathrm{~mm}$ cube.

To obtain the scaling factor during the tests with the mixer M2, both sizes of samples were taken 40x40x40 mm and 100x100x100 mm. Samples were kept in the same conditions and then tested for compressive strength. The obtained values were then compared and the average scaling factor of 1.15 was calculated.

Breaking time of the mix was noted as the time it takes for concrete to change from a dry state in the mixer to a fluid-like state, starting from the point at which water is added to the mix. This is measured with a precision of 30 seconds, to reduce the observer's bias in the recorded results. 


\section{Results and discussion}

The obtained results show that the differences in the mixer type led to notable changes in the observed relative viscosity of concrete. For all of the mixes except SP1-00, the relative viscosity increased, when the M2 mixer was used instead of the M1 mixer. This might be due to the increased mixing speed and the difference in the mixing method that might have led to an increase in the level of trapped air within the mix, which then leads to a reduction of the relative viscosity. Other researchers agree that an increase in the mixing speed will lead to a reduction in the yield stress and plastic viscosity of the mix [5;10]. It may be that the batch size also has an effect on the rheology of the mix and the efficiency of the mixing process, thus it should be further researched.

As it is shown in Fig. 4, the SP1 mix series showed a strong relationship between the relative viscosity and MS dosage when mixed in the M1 mixer, but not so much in the mixer M2. This suggests that both of the SPs react differently with cement and MS. The already higher viscosity of the SP1-00 mix might have been exaggerated by the mixer M1 and the increased friction that this type of mixer generates, thus generating more heat which leads to a further increase in the relative viscosity. The addition of MS to all of the mixes showed a clear reduction in the relative viscosity for both of the mixer types tested.

The mix SP2-40P showed a clear increase in the relative viscosity of the mix when the mixer M2 was used instead of the mixer M1. However, when mixing in the mixer M3, the results were comparable to the M1 results. These results indicate that the primary way of mixing has an effect on the relative viscosity of the mix.

Statistical analysis of the obtained data shows a moderately strong relationship between the relative viscosity of the mix, measured in seconds, and the resulting compressive strength of the UHPC mix shown in Fig. 5. The coefficient of determination value of the obtained model is 0.788 meaning that the obtained relation is moderately strong.

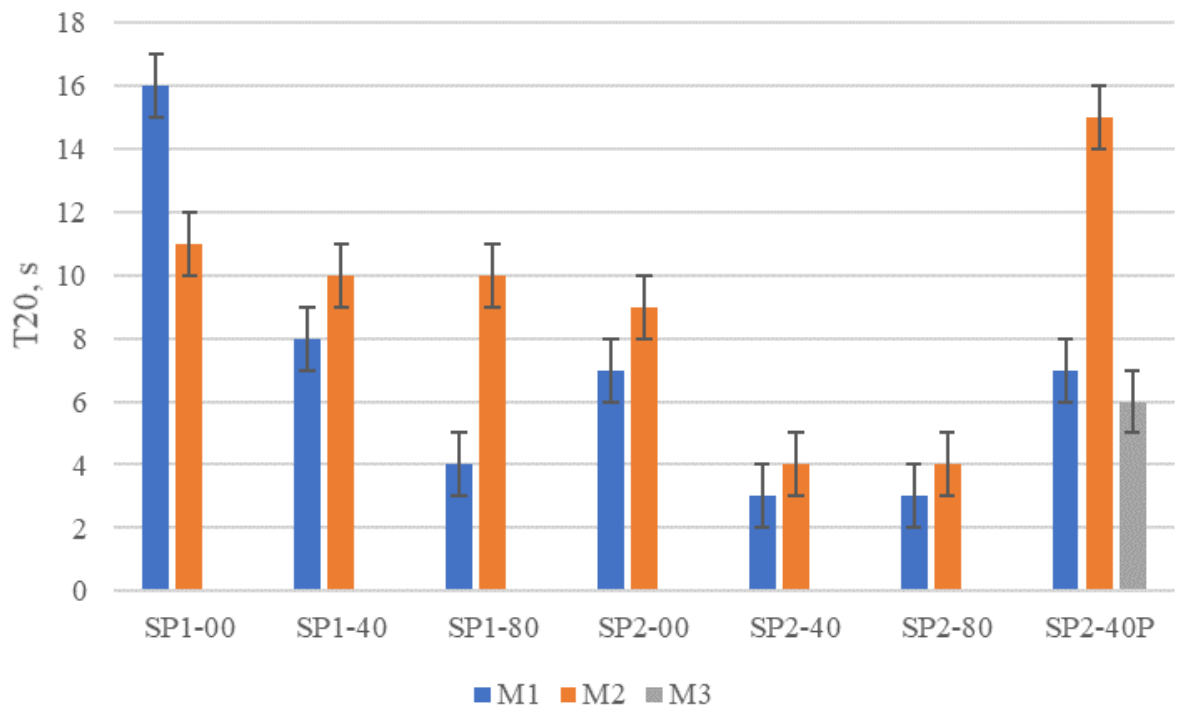

Fig. 4. Relative viscosity of the mix depending on the mixer used

Similarly as for relative viscosity measurements, the maximum slump flow of concrete mixed in the mixer M1 was generally higher than that of the concrete mixed in the mixer M2. Notable is the observation that the SP1 plasticizer was affected by the addition of MS much more than the SP2 plasticizer, which seemed not to be affected by the varying amount of MS as shown in Fig. 6. In addition, as in the relative viscosity measurements, mixers M1 and M3 behave similarly when used to mix SP2$40 \mathrm{P}$.

Analysis of the obtained data shows a mild correlation between the maximum slump flow obtained and the resulting compressive strength shown in Fig. 7. The coefficient of determination value of only 0.50 suggests further investigation in the effect the maximum slump flow of a particular UHPC mix has on the resulting compressive strength. 


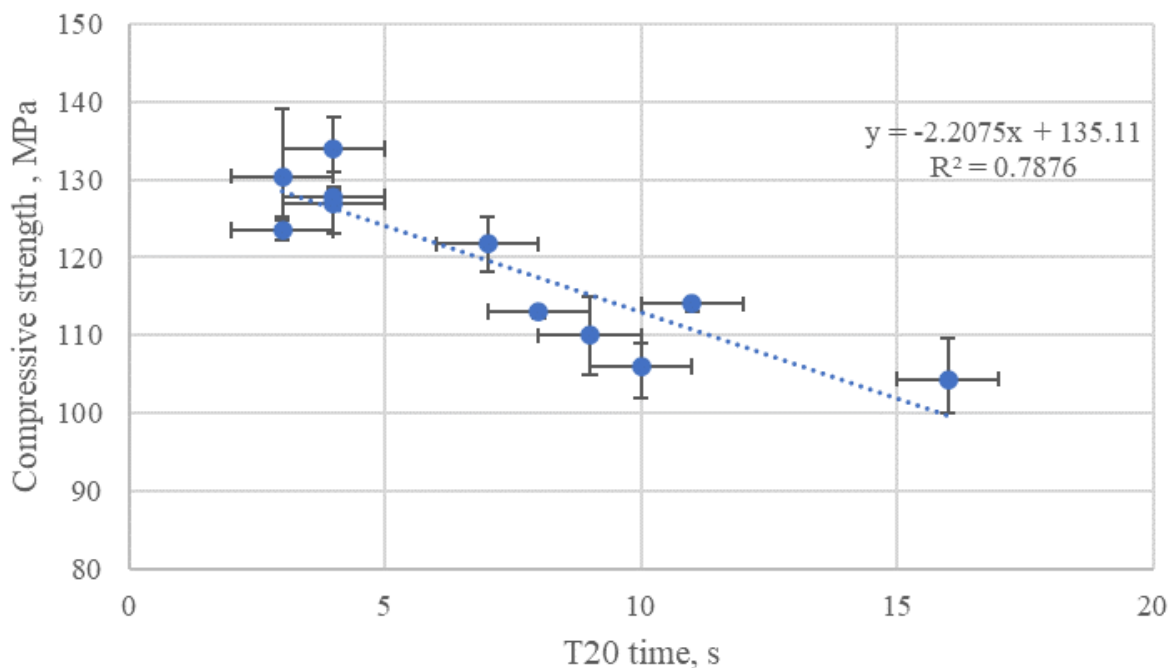

Fig. 5. Compressive strength dependency on the relative viscosity

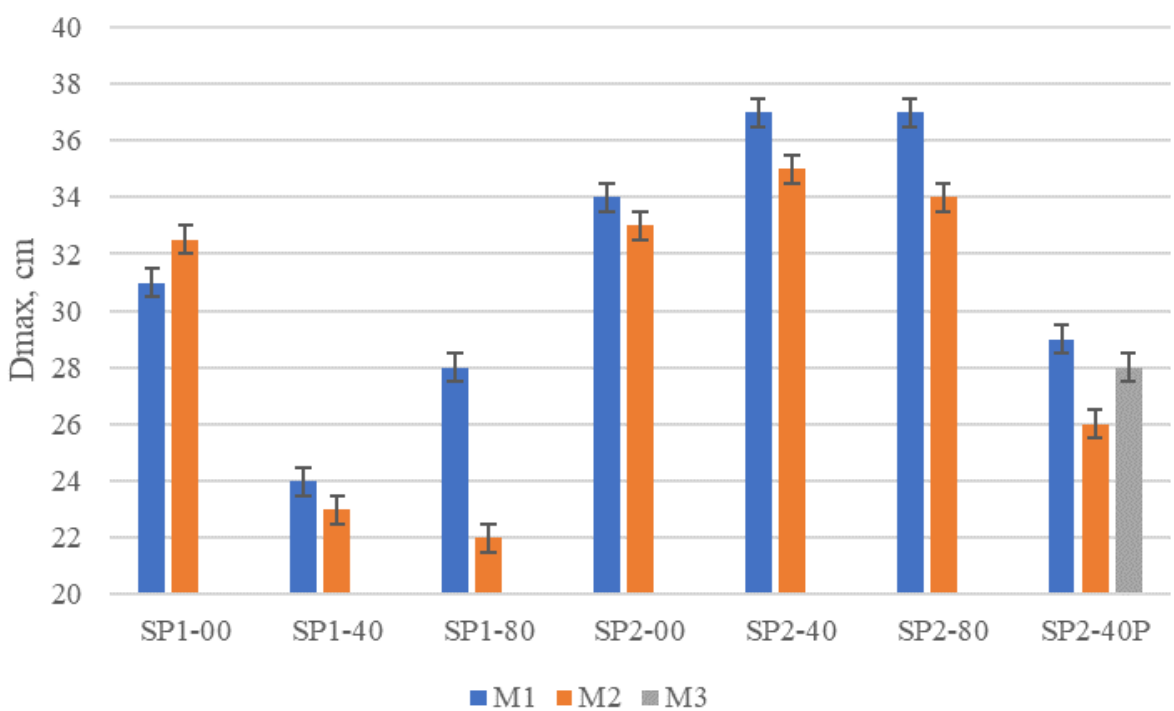

Fig. 6. Maximum slump flow of the mix depending on the mixer used

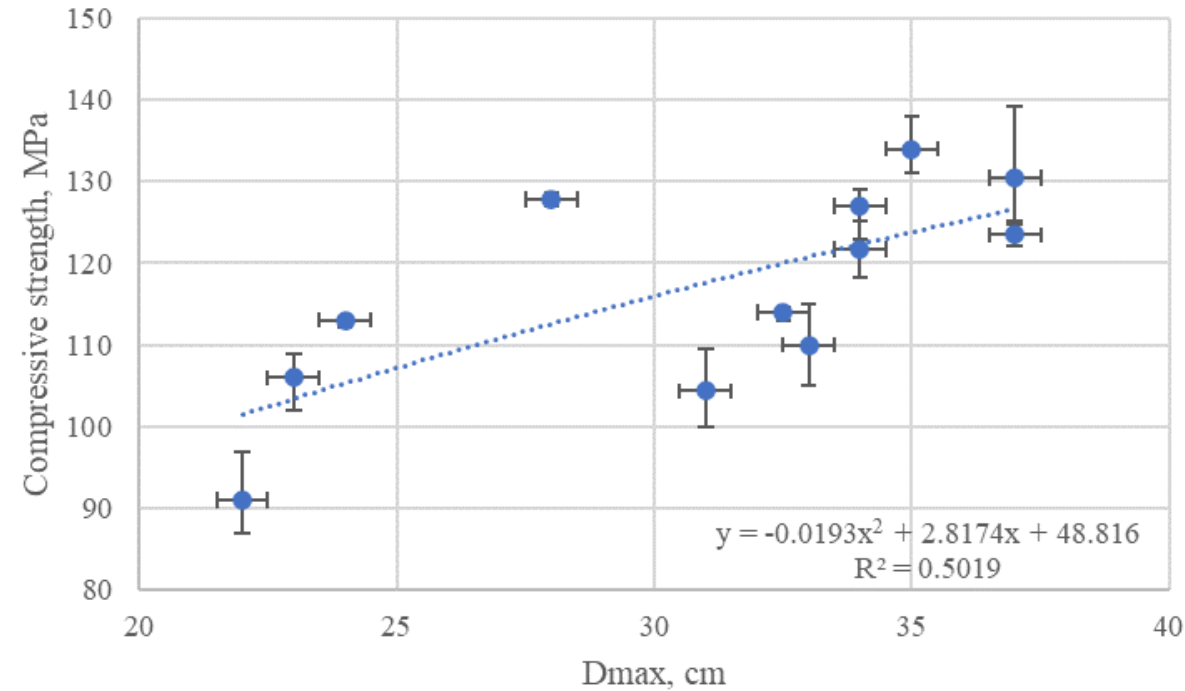

Fig. 7. Compressive strength dependency on the maximum slump flow

It has been shown in Fig. 8 that both types of mixers work in a slightly different way with the concrete mixes tested. Mixer M1 consistently showed a lower breaking time of the mix for the SP2 
plasticizer. This might be because the intensity of the mixing process and the batch size between both of the mixers are different. However, it could also be because of the increase in the entrapped air in the Hobart mixer leading to a lower yield stress of the mix being achieved at a shorter period of time, thus achieving the breaking point quicker.

For the SP1 plasticizer, a similar correlation between the breaking time of the mix and the mixer type has been observed. However, this relation does not follow that of the SP2 plasticizer. For the mix SP1-80, the breaking time increases. The initial decrease in the breaking time of the mix SP1-40 can be attributed to benefits from improved particle packing. These are then overcome by the increased surface area of the MS particles when MS dosage is further increased, thus increasing the breaking time of the mix. This is because the SP1 plasticizer has not been optimized for MS particle dispersion and only works by dispersing cement particles.

The breaking time seems to be significantly affected by the mixing speed and consequently mixing energy when two mixers of similar type are compared. The breaking time of the mix was significantly longer when mixed in mixer M3, although both of the mixers are of the pan mixer type.

The analysis of the obtained results indicates that some correlation between the breaking time of the mix, measured in minutes, and the obtained compressive strength can be made. The coefficient of determination value of the obtained model is only 0.58 , suggesting that further research is necessary. In Fig. 9 it can be seen that a reduction in the breaking time, in general, leads to an increase in the final compressive strength.

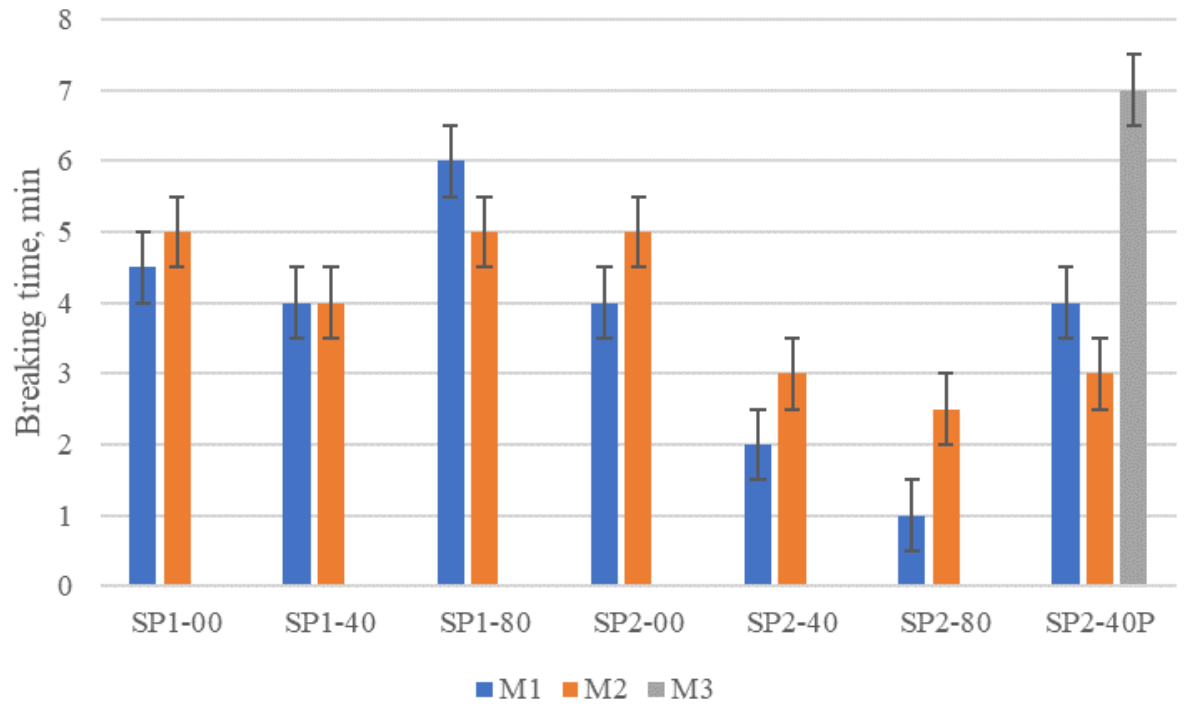

Fig. 8. Breaking time of the mix depending on the mixer used

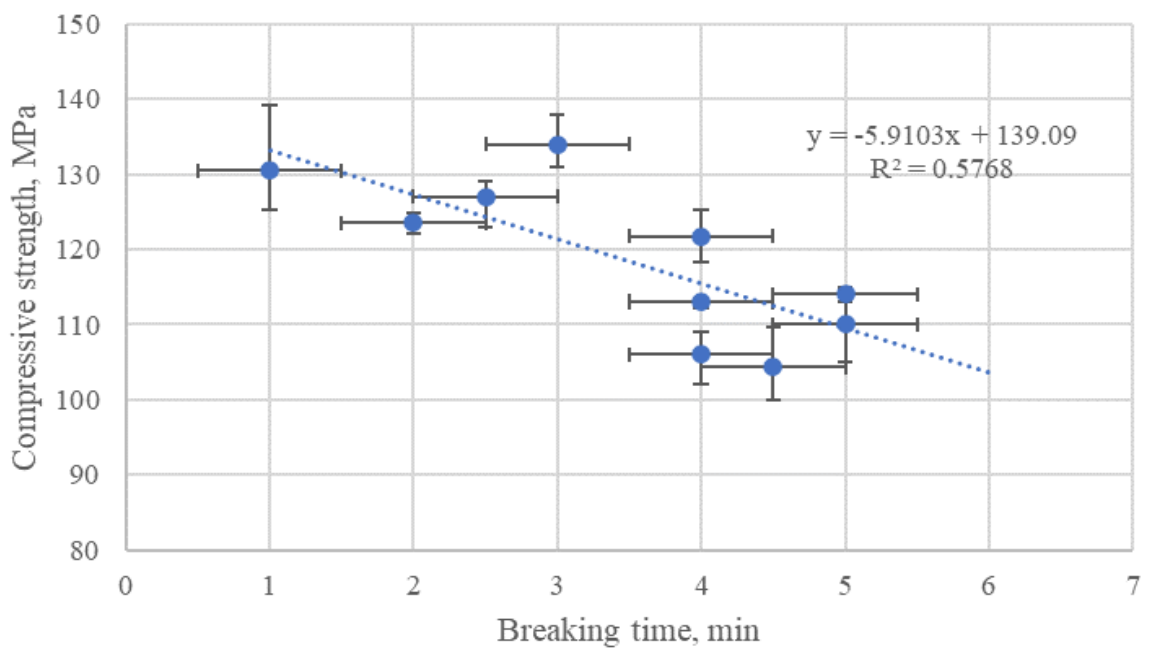

Fig. 9. Compressive strength dependency on the breaking time of the mix 
Fig. 10 shows that the 7-day compressive strength is higher for the samples mixed in the mixer M1, suggesting the mixer M1 is more effective. The mix SP1-00 is the only exception with higher strength when mixed in the mixer M2. This can be explained by the fact that SP1-00 also was the only mix that had better rheological parameters when mixed in the mixer M2. This led to better packing for the samples used for compressive strength testing.

It can also be noticed that plasticizer SP2 achieved much better homogeneity of the mix, as the compressive strength of the samples was much more consistent. For the plasticizer SP1, it can be seen that the addition of MS to the mix affects the homogeneity of the mix greatly regardless of the mixer and batch size.

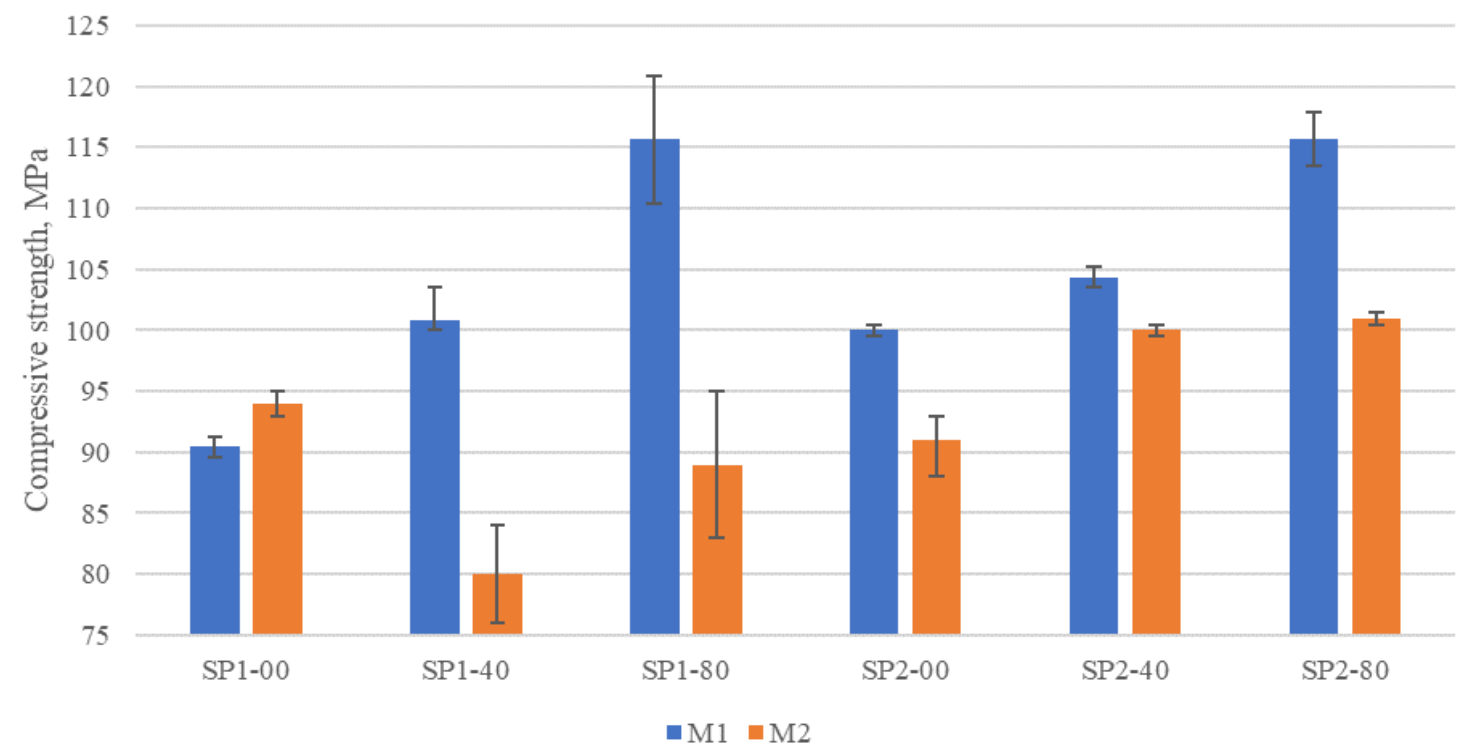

Fig. 10. 7-day compressive strength of concrete depending on the mixer used

The 28-day compressive strength results in Fig. 11 show increased strength with an increase in the MS dosage, when mixed in the mixer M1 for the SP1 plasticizer, however, the opposite is true when mixed in the mixer M2. This is not observed for the SP2 plasticizer. The reason behind this behaviour might be the increased mixing efficiency of the mixer M1. That allows for efficient MS particle distribution even when the SP does not help in dispersing them. However, when mixing in the mixer M2, there is a reliance on the SP to distribute the MS particles evenly. The reduction in the overall compressive strength thus may be explained by the reduction in the amount of cement within the mix and inefficient use of MS for strength gain and reduction in workability for the mix SP1-80.

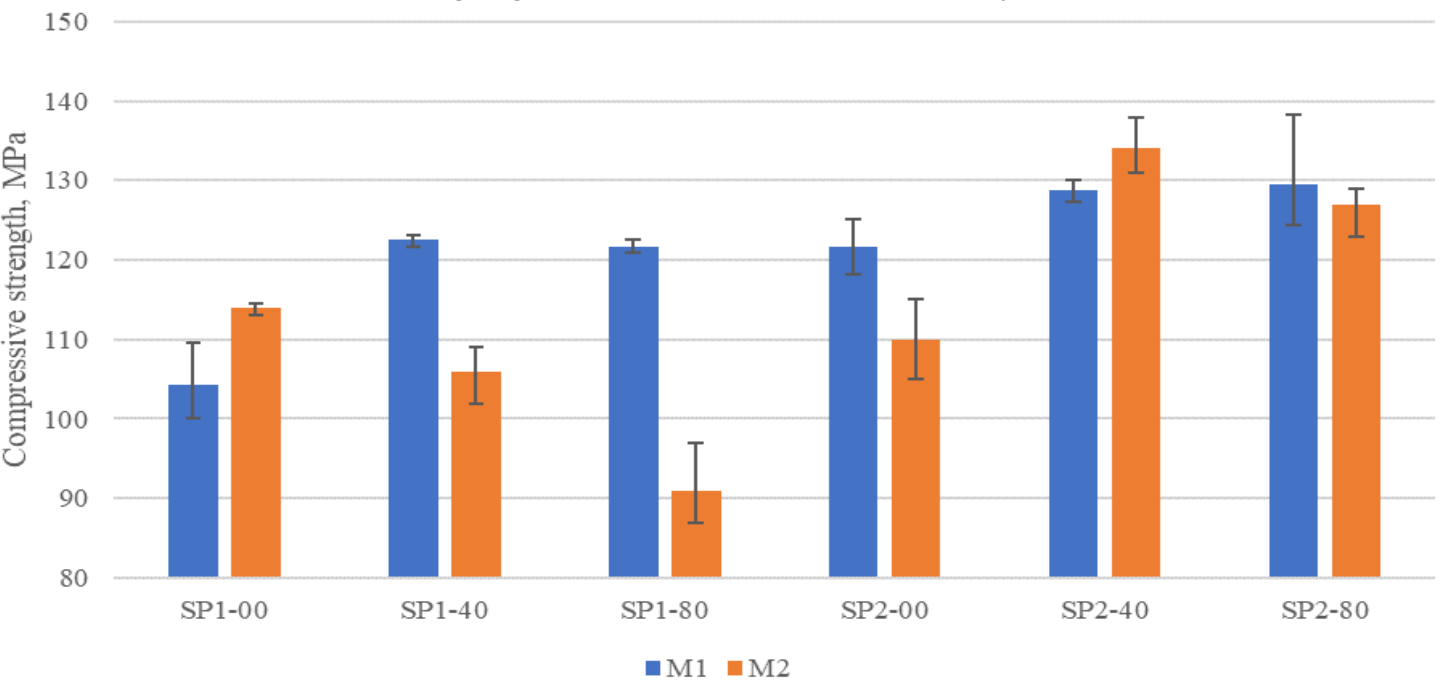

Fig. 11. 28-day compressive strength depending on the mixer used 
The results also indicate that overall much more consistent compressive strength results, thus more homogeneous mixture is achieved when using the mixer M1. The results from the mixer M2 show a greater scatter in the compressive strength test results. Previous research also suggests that improper mixing can lead to inhomogeneous UHPC mix [6;10].

For mixes with low relative viscosity (SP2-40 and SP2-80), the compressive strength of the samples mixed with both of the mixers is similar. However, when the relative viscosity of the mix is higher, differences between both mixers can be observed. For mixes with the SP1 superplasticizer the addition of micro silica resulted in a maximum increase of the compressive strength of $18.8 \%$, when UHPC was mixed in the mixer M1. However, when mixing in the mixer M2, the compressive strength was reduced by $20.2 \%$, showing how significantly a mixer can affect the compressive strength of UHPC.

\section{Conclusions}

1. More consistent compressive strength results are achieved in laboratory conditions, suggesting a more homogenous mixture is obtained.

2. Use of a pan mixer in a laboratory setting is advisable, as a pan mixer is also used for large scale manufacturing, thus no significant alteration to the mix design will be required when scaling up from laboratory to factory. The use of a drum mixer in a laboratory setting will require a significant alteration to the mix design when scaling up in the factory using a pan mixer.

3. Superplasticizer has to be optimized for the constituent materials in the UHPC mix, otherwise negative effects when adding micro silica or other cement replacement materials can be observed. In this study addition of micro silica resulted in an improvement of $18.8 \%$ for the compressive strength when mixed in the mixer M1. However, the same UHPC mix showed a reduction in the compressive strength by $20.2 \%$ when mixed in the mixer M2 when compared with a mix that has no micro silica added to it.

4. Rheology of fresh concrete was not significantly affected by the differences between the mixer M1 and M2, however, there were observable differences in the compressive strength between the samples made with either mixer.

5. When using superplasticizer SP2, the mixer M1 was more efficient at mixing concrete as the breaking time of the mix was at least a minute shorter when compared to the mixer M2. This is due to the increased mixing energy and speed of the mixer M1.

6. Further research has to be done to evaluate the batch size effect on the properties of fresh and hardened UHPC while using the same mixer.

7. There are differences between the compressive strength observed when using different mixers, this might be due to an increase in the entrapped air in concrete or a difference in the mixing efficiency between mixers. Further research has to be done to find out, if either scaling up the batch size or using a different type of mixer will lead to an increase in the air content of concrete.

\section{Acknowledgements}

In accordance with the contract No. 1.2.1.1/18/A/007 between "Competence centre of smart materials and technologies" Ltd. and the Central Finance and Contracting Agency, concluded on 23rd of April, 2019, the study is conducted by "Dzelzsbetons MB" Ltd. with support from the European Regional Development Fund (ERDF) within the framework of the project "Competence centre of smart materials and technologies".

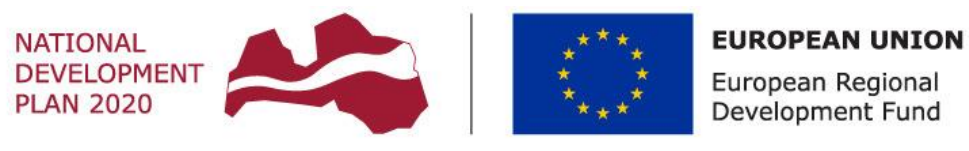

I N VESTING IN YOUR FUTURE

\section{References}

[1] Hiremath P., Yaragal S. Influence of mixing method, speed and duration on the fresh and hardened properties of Reactive Powder Concrete. Construction and Building Materials, vol. 141, 2017, pp. 271-288. 
[2] Federal Highway Administration, Ultra-High Performance Concrete: A State-of-the-Art Report for the Bridge Community. 2013.

[3] Schmidt M. Microstructural and performance based criteria for UHPC with improved durability outcome of a coordinated research programm in Germany. Marseille: Int. Symposium on UltraHigh Performance Fibre-reinforced Concrete, 2013, pp. 757-766.

[4] Wang R., Gao X., Zhang J., Han G. Spatial distribution of steel fibers and air bubbles in UHPC cylinder determined by X-ray CT method. Construction and Building Materials, vol. 160, 2018, pp. 39-47.

[5] Banfill P., Swift D. The effect of mixing on the rheology of cement-based materials containing high performance superplasticisers. Annual Transactions of the Nordic Rheology Society, vol. 12, 2004, pp. 9-12.

[6] Schaefer V., Wang K. Improving Portland cement concrete mix consistency and production rate through two-stage mixing. Iowa State University. 2007.

[7] Ferraris C. Concrete Mixing Methods and Concrete Mixers: State of the Art. Journal of Research of the National Institute of Standards and Technology, vol. 106(2), 2001, pp.391-399.

[8] Tackett A., Hale W. Examining the effects of mixer type and temperature on the properties of ultrahigh performance concrete. n.d.

[9] Li H., Huang F., Yi Z., Wang Z., Zhang Y., Yang, Z. Investigations of Mixing Technique on the Rheological Properties of Self-Compacting Concrete. Applied Sciences, vol. 10(15), 2020, p.5189.

[10] Dehn F. Influence of mixing technology on fresh concrete properties of HPFRCC. Proceedings of Int'l RILEM workshop on HPFRCC in structural applications, 2006.

[11]Zavickis J., Lukasenoks A., Macanovskis A., Tupesis M. Optimization of packing of local coarse aggregates for use in UHPC (Ultra-High-Performance Concrete). 19th International Scientific Conference Engineering for Rural Development Proceedings, 2020. 